\title{
Characterization and Quantification of Luteolin-Metal Complexes in Aqueous Extract of Lonicerae Japonicae Flos and Huangshan Wild Chrysanthemum
}

\author{
Weilan Cai, Yunhao Xiong, Manman Han, Zhimin Li $\mathbb{D}$, Liang Peng $\mathbb{D}^{\text {, }}$, Hongyi Zhang, \\ Qin Zou, Lin Wu, Qingling Ye, and Linfeng Liao
}

Jiangxi Provincial Key Laboratory of Drug Design and Evaluation, School of Pharmacy, Jiangxi Science \& Technology Normal University, Nangchang, Jiangxi 330013, China

Correspondence should be addressed to Zhimin Li; xingzan22@163.com and Liang Peng; lunwang703@163.com

Received 3 November 2020; Revised 8 February 2021; Accepted 1 March 2021; Published 12 March 2021

Academic Editor: Valentina Venuti

Copyright (c) 2021 Weilan Cai et al. This is an open access article distributed under the Creative Commons Attribution License, which permits unrestricted use, distribution, and reproduction in any medium, provided the original work is properly cited.

\begin{abstract}
Luteolin is a flavonoid compound widely found in vegetables, fruits, and medicinal plants. In this study, the reaction conditions for luteolin and five metal ions $\left(\mathrm{Ca}^{2+}, \mathrm{Mg}^{2+}, \mathrm{Zn}^{2+}, \mathrm{Fe}^{3+}\right.$, and $\left.\mathrm{Cu}^{2+}\right)$ to form complexes in hot water were optimized, which was at a molar ratio of $1: 1$ for luteolin and metal ions at $90^{\circ} \mathrm{C}$ in a volume of $20 \mathrm{~mL}$ for $2 \mathrm{~h}$, and the ability of luteolin to form complexes with $\mathrm{Cu}^{2+}$ was the strongest. The DPPH scavenging test showed that luteolin exerted a dose-dependent effect on the clearance of free radicals; luteolin- $\mathrm{Cu}^{2+}$ complexes and luteolin- $\mathrm{Fe}^{3+}$ complexes accentuated the clearance of free radicals. Furthermore, we used high performance liquid chromatography (HPLC) to analyze luteolin in samples from two medicinal plants, obtained from the dissolution of aqueous extracts in two different solvents. The results showed that the peak areas for luteolin in the samples dissolved in $20 \%$ formic acid-methanol were significantly larger than those from the samples dissolved in methanol alone, with increases in the peak area being 135.6\% (Lonicerae Japonicae Flos), and 161.16\% (Huangshan wild chrysanthemum). The aforementioned results indicate that complexes formed from organic compounds and metal ions are present in the decoction of a plant.
\end{abstract}

\section{Introduction}

Plants are an important source of many biologically active and clinically relevant organic compounds, including phenylpropanoid, flavonoid, anthraquinone, steroids, terpenoids, and alkaloids [1]. They also contain many metal ions such as calcium, magnesium, iron, and copper that form complexes with organic compounds which perform multiple biological activities [2]. Phenolic hydroxyl, carbonyl, carboxyl, sulfhydryl, and amino groups and nitrogen-containing aromatic rings are nearly universally present in organic compounds and may readily form complexes with metals. Rybak and Ruzik [3] found that manganese formed complexes with rutin, alizarin, and asperulosidic acid in noni juice. Using $\mu \mathrm{HPLC}-\mathrm{ESI}-\mathrm{MS} / \mathrm{MS}$, Wojcieszek et al. [4] detected seven types of copper complexes and four types of zinc complexes in ionic liquid and pectinase extracts of Lycium barbarum. Weber and Konieczyński [5] detected manganese and magnesium complexes in Folium Betulae (birch leaves), Folium Menthae (peppermint leaves), and Radix Taraxaci (dandelion roots) by size-exclusion chromatography combined with atomic absorption spectroscopy. Using the membrane filter and macroporous resin, Liu et al. [6] identified by flame atomic absorption spectrometry many complexes between zinc and proteins or polycarbohydrates in the decoction of Flammulina velutipes. These studies indicated that multiple complexes of ions and organic compounds are present in plants in nature.

Luteolin is a flavonoid that is widely present in nature and is mainly distributed in medicinal herbs such as Lonicerae Japonica Flos, Origanum vulgare, chrysanthemum, and rosemary and in foods including peanuts, carrots, celery, cucumber, and buckwheat. $[7,8]$ Luteolin possesses 
many biological activities including antioxidant $[9,10]$, antibacterial [11], antiviral [12], and antitumor activities $[13,14]$ and also confers cardiocerebrovascular protection $[15,16]$. The phenolic hydroxyl and carbonyl groups have intact $\pi$ conjugation and strong superdelocalizability and proper planar structure, which facilitate complex formation between luteolin and ions.

In the current study, we investigated luteolin ion complex formation by measuring postreaction luteolin content by high performance liquid chromatography (HPLC). We further determined the effects of reaction temperature, volume, and time on the formation of complexes between luteolin and five types of ion including calcium, magnesium, iron, zinc, and copper in the aqueous extract. We also studied the effects of ions on free radical scavenging activities of luteolin. Finally, we determined the presence of luteolin ion complexes in Lonicerae Japonica Flos (L. japonica) and Huangshan wild chrysanthemum by HPLC analysis of aqueous extract of $L$. japonica and Huangshan wild chrysanthemum in methanol and $20 \%$ formic acid-methanol.

\section{Materials and Methods}

2.1. Chemicals. Luteolin was purchased from Haochen Biotechnology Co. (Shaanxi, China), and its purity was confirmed to be greater than $98 \%$ by HPLC. Luteolin standard was purchased from the National Institute for the Control of Pharmaceutical and Biological Products (Beijing, China). Methanol (HPLC grade and mass spectrometry grade) was purchased from Merker, Germany; formic acid (HPLC grade) and deuterated methanol were purchased from Aladdin, USA, and 1,1-dipheny-2-trinitrophenylhydrazine (DPPH) was purchased from TCI Chemicals (Shanghai, China). Magnesium chloride hexahydrate, anhydrous calcium chloride, anhydrous zinc chloride, iron chloride hexahydrate, copper chloride dihydrate, ethyl acetate, and DMSO were all analytical grade. Weight of chemicals used was measured using an XS203S electronic scale (METTLER TOLEDO, Swiss).

2.2. Plants. L. japonica and Huangshan wild chrysanthemum were purchased from Yifeng Pharmacy (Nanchang, China). L. japonica was grown in Henan Province, China, and Huangshan wild chrysanthemum was grown in Anhui Province, China. They were officially authenticated by the School of Pharmacy, Jiangxi Science and Technology Normal University. L. japonica was dried flower buds of Lonicera japonica Thunb., and Huangshan wild chrysanthemum was the dried capitulum of Dendranthema morifolium (Ramat) Tzvel. cv. Gongju.

2.3. Preparation of Luteolin Standard Curve. HPLC separations were performed on YMC-Pack ODS-A C18 chromatographic column $(4.6 \mathrm{~mm} \times 150 \mathrm{~mm}, 5 \mu \mathrm{m})$ with $30^{\circ} \mathrm{C}$ column temperature. Luteolin was eluted using $2 \mathrm{mmol} \mathrm{L}^{-1}$ ammonium formate solution ( $0.1 \%$ formic acid) (A) and methanol (B) $(60: 40, \mathrm{~V} / \mathrm{V})$ as the mobile phase with $60 \% \mathrm{~B}$ isocratic elution. The flow rate was $1.0 \mathrm{~mL} \cdot \mathrm{min}^{-1}$. The injection volume was $1 \mu \mathrm{L}$. The wavelength of the VWD detector was set at $350 \mathrm{~nm}$.

Luteolin methanol stock solution $(2.5 \mathrm{mmol} / \mathrm{L})$ was prepared and diluted into the following concentrations: 2.25 , $2.0,1.75,1.5,1.25,1.0,0.75,0.5$, and $0.25 \mathrm{mmol} / \mathrm{L}$. The peak area of HPLC was determined. Luteolin standard curve was plotted with the HPLC peak area as the $x$-axis and luteolin concentrations as the $y$-axis. The following regression equation was derived:

$$
Y=0.001 X+0.0008(R=0.9999)
$$

indicating that luteolin concentration from $0.25 \mathrm{mmol} / \mathrm{L}$ to $2.5 \mathrm{mmol} / \mathrm{L}$ had a good linear relationship.

2.4. ${ }^{1} H-N M R$. Luteolin $(0.1 \mathrm{mmol})$ was allowed to react with $0.02 \mathrm{mmol}$ anhydrous calcium chloride, anhydrous zinc chloride, magnesium chloride hexahydrate, iron chloride hexahydrate, or copper chloride dihydrate in $40 \mathrm{~mL}$ distilled water at $90^{\circ} \mathrm{C}$ for $4 \mathrm{~h}$ under heat backflow using a Hei-VAP rotary evaporator (Heidolph, Germany) and mixed with a DF-101S heat collection type isothermal magnetic heating stirrer (Yuhua Co., Gongyi, Henan, China). Thereafter, the reactants were spun dry and washed with distilled water several times to remove ions that did not form complexes with luteolin. The remaining solid portion was washed several times with $100 \mathrm{~mL}$ ethyl acetate, and after the solid portion was spun dry, it was dissolved in deuterated methanol. Free luteolin and the reactants in deuterated methanol were then studied by nuclear magnetic resonance (NMR) spectroscopy as routinely performed using a Bruker AVANCE 400-MHz spectrometer.

2.5. Determination of Reaction Conditions. To determine the effect of reaction temperature on luteolin ion salt complex formation, we mixed $0.1 \mathrm{mmol}$ luteolin with $0.02 \mathrm{mmol}$ anhydrous calcium chloride, anhydrous zinc chloride, magnesium chloride hexahydrate, iron chloride hexahydrate, or copper chloride dihydrate in $40 \mathrm{~mL}$ distilled water. The reaction was allowed to proceed for $4 \mathrm{~h}$ under heat backflow at a temperature of $30^{\circ} \mathrm{C}, 50^{\circ} \mathrm{C}, 70^{\circ} \mathrm{C}$, or $90^{\circ} \mathrm{C}$. Furthermore, to determine the effect of reaction time on luteolin ion salt complex formation, we carried out the above reaction at $90^{\circ} \mathrm{C}$ for $0.5,1,2$, and $4 \mathrm{~h}$, respectively. In addition, to determine the effect of reaction volume on luteolin ion salt complex formation, we carried out the above reaction in $20,40,80$, or $160 \mathrm{~mL}$ distilled water at $90^{\circ} \mathrm{C}$ for $2 \mathrm{~h}$. We also investigated the effect of molar ratios of luteolin to ion salts on luteolin ion salt complex formation at a ratio of $10: 1,5: 1,2: 1$, and $1: 1(0.1 \mathrm{mmol}$ luteolin added to 0.002 , $0.004,0.01$, and $0.02 \mathrm{mmol}$ anhydrous calcium chloride, anhydrous zinc chloride, magnesium chloride hexahydrate, iron chloride hexahydrate, or copper chloride dihydrate, respectively). The reaction was allowed to proceed for $2 \mathrm{~h}$ under heat backflow at $90^{\circ} \mathrm{C}$. Finally, we examined the ability of luteolin to complex with different ion salts. Luteolin $(0.1 \mathrm{mmol})$ was allowed to react with $0.1 \mathrm{mmol}$ anhydrous calcium chloride, anhydrous zinc chloride, magnesium 
chloride hexahydrate, iron chloride hexahydrate, or copper chloride dihydrate in $20 \mathrm{~mL}$ distilled water at $90^{\circ} \mathrm{C}$ under heat backflow for $2 \mathrm{~h}$. Free luteolin without ion salt served as blank control.

The reactants were spun dry and washed with distilled water several times to remove ions that did not form complexes with luteolin. The remaining solid portion was washed several times with $100 \mathrm{~mL}$ ethyl acetate, and after the ethyl acetate lotion was spun dry, it was dissolved in $20 \mathrm{~mL}$ methanol. Luteolin content was analyzed by HPLC as described above.

2.6. DPPH Scavenging Test. Luteolin $(0.1 \mathrm{mmol})$ was allotted to react with $0.1 \mathrm{mmol}$ anhydrous calcium chloride, magnesium chloride hexahydrate, iron chloride hexahydrate, or copper chloride dihydrate in $20 \mathrm{~mL}$ distilled water at $90^{\circ} \mathrm{C}$ under heat backflow for $2 \mathrm{~h}$. The reactants were spun dry and dissolved in DMSO in $10 \mathrm{mmol} / \mathrm{L}$ stock solution (using the molar concentration of luteolin). Luteolin and the 4 ion salts were also prepared in equivalent molar concentration. Radical scavenging capacity was evaluated by a DPPH radical test as previously described [17]. The experiments were performed three times independently in triplicate, and the mean value was used to calculate clearance using the following equation:

$$
\text { Clearance }(\%)=\left(1-\frac{A_{s}-A_{c}}{A_{0}}\right) \times 100 \%,
$$

where As represents the mean absorbance of the sample and $\mathrm{DPPH}, \mathrm{Ac}$ represents the mean absorbance value of the sample, and $A_{0}$ represents the mean absorbance value of DPPH solution.

2.7. HPLC Analysis. L. japonica and Huangshan wild chrysanthemum $(20 \mathrm{~g}$ each) were dissolved in $200 \mathrm{~mL}$ distilled water and after soaking at room temperature for $30 \mathrm{~min}$, were subjected to heat backflow decoction at $95^{\circ} \mathrm{Cfor}$ $2 \mathrm{~h}$. After filtration, the filtrate was spin-dried to obtain dried decoction. The dried decoction was dissolved in $20 \mathrm{~mL}$ methanol or $20 \mathrm{~mL} 20 \%$ formic acid-methanol, and after sonication for $1 \mathrm{~min}, 1 \mathrm{~mL}$ solute was filtered using $0.22 \mu \mathrm{m}$ membrane and then subjected to HPLC.

HPLC separations were performed on an Agilent Eclipse XDB-C18 analytical column $(250 \mathrm{~mm} \times 4.6 \mathrm{~mm}, 5 \mu \mathrm{m})$ with $30^{\circ} \mathrm{C}$ column temperature. Samples were eluted using $2 \mathrm{mmol} \mathrm{L}^{-1}$ ammonium formate solution ( $0.2 \%$ formic acid or methanol) (A) and acetonitrile (B) $(60: 40, \mathrm{~V} / \mathrm{V})$ as the mobile phase $0-80 \mathrm{~min}, 20 \% \mathrm{~B} \longrightarrow 60 \% \mathrm{~B}$; $80-100 \mathrm{~min}, 60 \%$ $\mathrm{B} \longrightarrow 100 \% \mathrm{~B}$. The flow rate was $0.2 \mathrm{~mL} \cdot \mathrm{min}^{-1}$. The injection amount was $10 \mu \mathrm{L}$. The wavelength of the DAD detector was set at $350 \mathrm{~nm}$.

\section{Results}

3.1. Effect of Reaction Temperature on Luteolin-Metal Complex Formation. ${ }^{1} \mathrm{H}-\mathrm{NMR}$ spectroscopy showed that after its reaction with anhydrous calcium chloride, magnesium chloride hexahydrate, iron chloride hexahydrate, or copper chloride dihydrate, luteolin complexes in ethyl acetate exhibited similar spectra to those of free luteolin (Figure 1), indicating that luteolin did not completely react with the five types of ion salts, and luteolin ion salt complexes were not dissolvable in ethyl acetate. Therefore, in subsequent experiments, HPLC was used to quantify luteolin content in ethyl acetate, with higher luteolin content in ethyl acetate indicating a lower capacity to form complexes with these ions.

We then allowed luteolin to react with anhydrous calcium chloride, magnesium chloride hexahydrate, iron chloride hexahydrate, or copper chloride dihydrate at different temperatures to investigate the effect of reaction temperature on luteolin ion complex formation. We observed a gradual reduction in the peak area of luteolin as the reaction temperature rose from $30^{\circ} \mathrm{C}$ to $90^{\circ} \mathrm{C}$ (Figure 2). At $90^{\circ} \mathrm{C}$, the peak area of luteolin was $67.2 \%$ of that at $30^{\circ} \mathrm{C}$. The reaction temperature was set at $90^{\circ} \mathrm{C}$ for subsequent experiments.

3.2. Effect of Reaction Time and Volume on Luteolin-Metal Complex Formation. We also delineated the effect of reaction time on luteolin ion complex formation. The amount of luteolin gradually decreased as the reaction time increased (Figure 3(a)). At $4 \mathrm{~h}$, the peak area of luteolin decreased by $9.7 \%$ versus that at $2 \mathrm{~h}$, suggesting that most luteolin ion complexes had already formed by $2 \mathrm{~h}$. The reaction time was set at $2 \mathrm{~h}$ for subsequent experiments. We further studied the effect of solvent volume on luteolin ion complex formation. We observed no apparent difference in the content of luteolin in ethyl acetate among different reaction volumes (Figure 3(b)). The peak area at a volume of $40 \mathrm{~mL}$ was the smallest and declined only by $1.98 \%$ versus that at a volume of $20 \mathrm{~mL}$, which fell within the standard error of weighing. The peak area at a volume of $80 \mathrm{~mL}$ and $160 \mathrm{~mL}$ increased only by $6.00 \%$ and $9.04 \%$, respectively, compared with that at a volume of $20 \mathrm{~mL}$. This could be due to lower ion concentrations with increased volume, which does not facilitate complexation. The reaction volume was set at $20 \mathrm{~mL}$ for subsequent experiments.

\subsection{Effect of Molar Ratios of Luteolin to Ions and Different} Types of Ions on Luteolin Ion Complex Formation. Luteolin was added at a molar ratio of $10: 1,5: 1,2: 1$, or $1: 1$ to ions. As shown in Figure 4(a), when the molar ratio of luteolin to ions was $1: 1$, the amount of luteolin in ethyl acetate was only $31.8 \%$ of the amount of luteolin when the luteolin to ion ratio was $10: 1$, suggesting that the amount of ions had a marked effect on luteolin ion complex formation: the greater the amount of ions was, the greater the amount of luteolin ion complexes was formed. In subsequent studies, the ratio of luteolin to ions was maintained at $1: 1$.

We further examined luteolin ion complex formation between luteolin and different types of ions. Under condition for water decoction at $90^{\circ} \mathrm{C}$ under heat backflow for $2 \mathrm{~h}$, the peak area was the smallest following luteolin and $\mathrm{Cu}^{2+}$ reaction (Figure 4(b)). The peak area after luteolin and $\mathrm{Zn}^{2+}$ reaction increased $1.82 \%$ compared to that with no ions, 


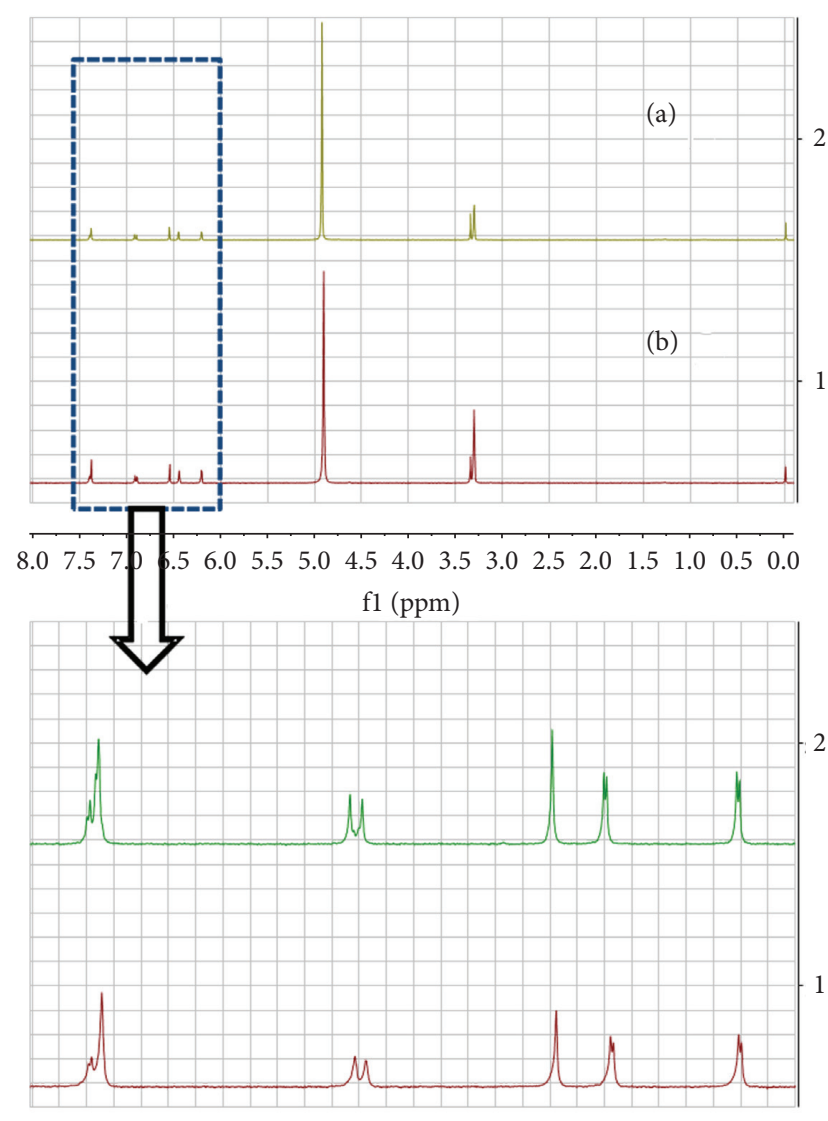

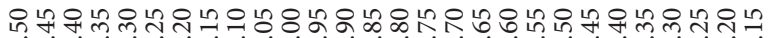

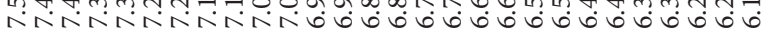
f1 (ppm)

FIGURE 1: Luteolin was allowed to react with anhydrous calcium chloride, anhydrous zinc chloride, magnesium chloride hexahydrate, iron chloride hexahydrate, or copper chloride dihydrate. After removal of free ion salts, the reactants were analyzed by ${ }^{1} \mathrm{H}-$ NMR spectroscopy. (a) Free luteolin was used as a control; (b) ethyl acetate solute.

indicating virtually no formation of the luteolin- $\mathrm{Zn}^{2+}$ complex. The ability to form luteolin ion complexes was as follows: $\mathrm{Cu}^{2+}>\mathrm{Fe}^{3+}>\mathrm{Ca}^{2+}>\mathrm{Mg}^{2+}>\mathrm{Zn}^{2+}$.

3.4. Effect of Metal Ions on Clearance of Free Radicals by Luteolin. Luteolin exhibited a dose-dependent effect on clearance of free radicals. The clearance rate of free radicals was $18.34 \%$ for luteolin at $0.10 \mathrm{mmol}$, which shot up to $80.27 \%$ for luteolin at $1.0 \mathrm{mmol}$ (Table 1). The clearance rate of free radicals stood at $89.69 \%, 73.44 \%, 94.63 \%$, and $79.46 \%$ for luteolin- $\mathrm{Fe}^{3+}$ complexes, luteolin- $\mathrm{Mg}^{2+}$ complexes, luteolin- $\mathrm{Cu}^{2+}$ complexes, and luteolin- $\mathrm{Ca}^{2+}$ complexes at $1.0 \mathrm{mmol} / \mathrm{L}$, respectively. In the absence of luteolin, the clearance rate of free radicals was 29.81\%, 7.0\%, 39.39\%, and 5.70\% for $\mathrm{Fe}^{3+}, \mathrm{Mg}^{2+}, \mathrm{Cu}^{2+}$, and $\mathrm{Ca}^{2+}$ salt only at $1.0 \mathrm{mmol} / \mathrm{L}$, respectively. These findings suggested that luteolin- $\mathrm{Cu}^{2+}$ complexes and luteolin- $\mathrm{Fe}^{3+}$ complexes accentuated the clearance of free radicals.

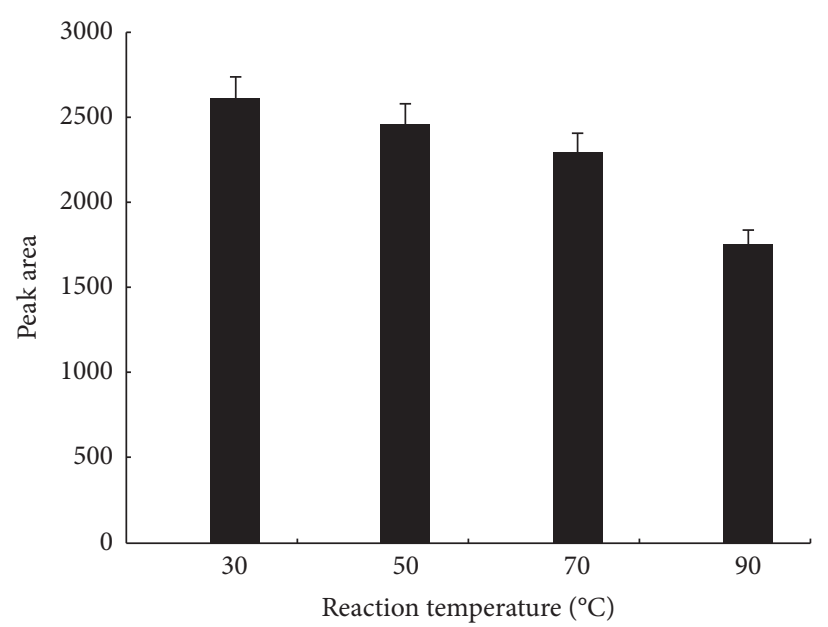

FIGURE 2: Effect of reaction temperature on luteolin-metal complex formation. Luteolin $(0.1 \mathrm{mmol})$ was mixed with $0.02 \mathrm{mmol}$ anhydrous calcium chloride, anhydrous zinc chloride, magnesium chloride hexahydrate, iron chloride hexahydrate, or copper chloride dihydrate in $40 \mathrm{~mL}$ distilled water. The reaction was allowed to proceed for $4 \mathrm{~h}$ under heat backflow at a temperature of $30^{\circ} \mathrm{C}, 50^{\circ} \mathrm{C}$, $70^{\circ} \mathrm{C}$, or $90^{\circ} \mathrm{C}$. After free ion salts were removed, the reactants were analyzed by HPLC as described in methods. The experiment was performed at least three times independently.

\subsection{Luteolin-Metal Complexes in L. japonica and Huangshan} Wild Chrysanthemum Decoction. The chromatographic profiles of the methanol extract and $20 \%$ formic acid-methanol extract of L. japonica decoction are shown in Figure 5, and the retention time and peak area of ten well-isolated chromatographic peaks are given Table 2 . The chromatographic profiles of the methanol extract and $20 \%$ formic acid-methanol extract of Huangshan wild chrysanthemum decoction are shown in Figure 6 , and the retention time and peak area of ten wellisolated chromatographic peaks are given Table 3.

The retention time of the methanol extract of L. japonica decoction was $60.26 \pm 0.02 \mathrm{~min}$, and the peak area was $1091.53 \pm 13.15$. The retention time of the $20 \%$ formic acid-methanol extract of $L$. japonica decoction was $60.24 \pm 0.02 \mathrm{~min}$, and the peak area was $1094.37 \pm 9.28$. Luteolin contains multiple phenolic hydroxyl groups and is weakly acidic, which, to a certain extent, modifies its chromatographic behavior; therefore, in the $20 \%$ formic acid-methanol extract, the peak appeared earlier than that in the methanol extract. However, there was no difference in the peak area between the two extracts.

The chromatographic profiles of the methanol extract and $20 \%$ formic acid-methanol extract of L. japonica decoction and Huangshan wild chrysanthemum decoction were largely in agreement with one another (Figures 5 and 6). In Huangshan wild chrysanthemum decoction, the number of chromatographic peaks, resolution, and peak form were identical in the two extracts. L. japonica decoction had two apparent chromatographic peaks at $55.249 \mathrm{~min}$ and $56.273 \mathrm{~min}$ in the $20 \%$ formic acid-methanol sample. Though no apparent chromatographic peaks were observed 


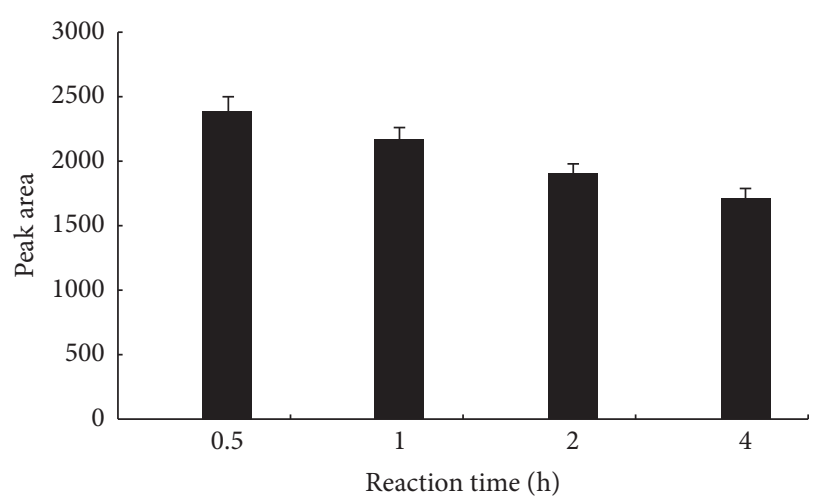

(a)

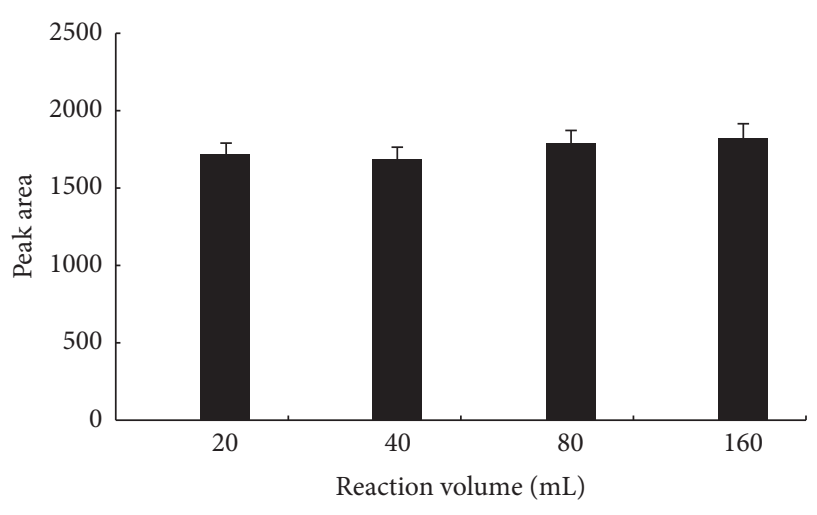

(b)

Figure 3: Effects of reaction time and volume on luteolin-metal complex formation. (a) Luteolin (0.1 mmol) mixed with $0.02 \mathrm{mmol}$ anhydrous calcium chloride, anhydrous zinc chloride, magnesium chloride hexahydrate, iron chloride hexahydrate, or copper chloride dihydrate was allowed to proceed for $0.5,1,2$, or $4 \mathrm{~h}$ under heat backflow at a temperature of $90^{\circ} \mathrm{C}$. (b) The reaction in (a) was carried out in $20,40,80$, or $160 \mathrm{~mL}$ distilled water at 90 for $2 \mathrm{~h}$. After free ion salts were removed, the reactants were analyzed by HPLC as described in methods. The experiment was performed at least three times independently in triplicate.

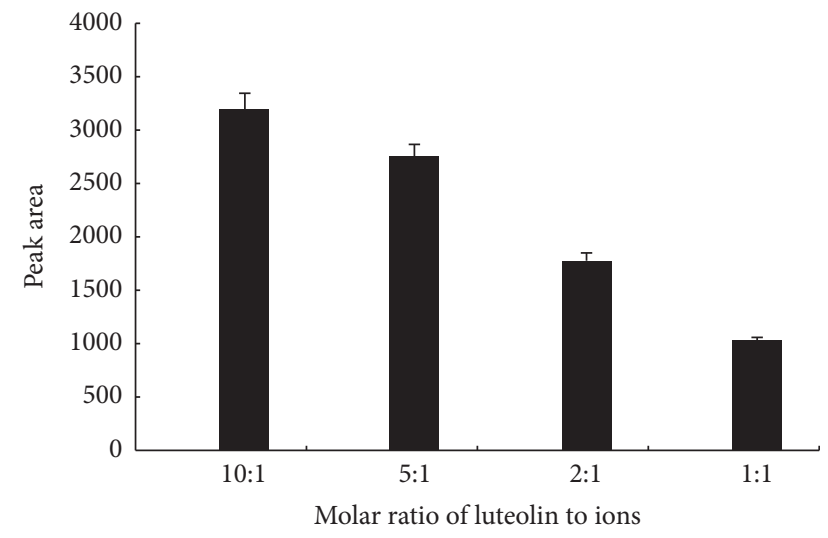

(a)

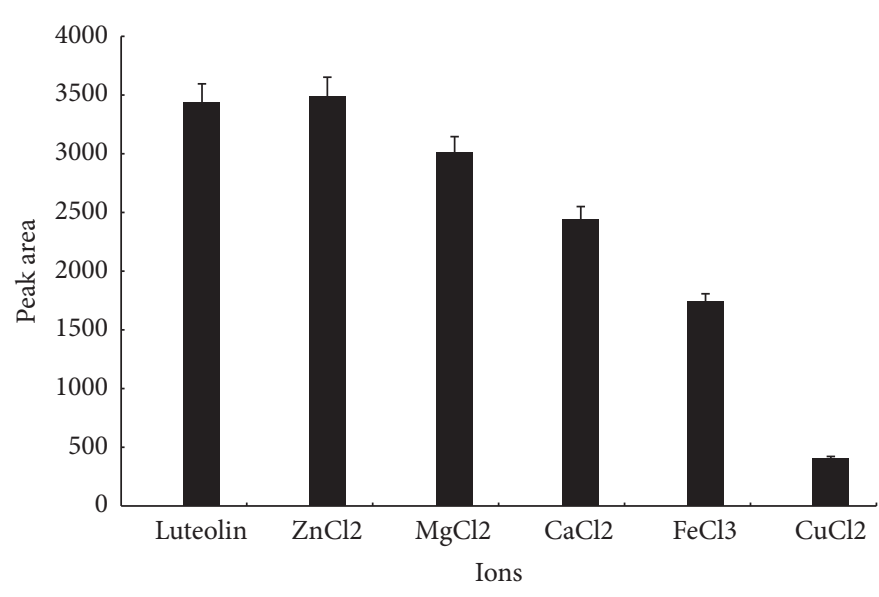

(b)

FIGURE 4: (a) Effect of the molar ratio of luteolin to metal ions on the formation of luteolin-metal complex ( $n=3)$. (b) Effect of different types of metal ions on luteolin-metal complex formation $(n=3)$.

TABLE 1: Effect of luteolin, metal salt and luteolin, and metal ion reaction products on clearance of DPPH free radicals.

\begin{tabular}{lccccccccc}
\hline \multirow{2}{*}{ Concentration, mmol/L } & & \multicolumn{9}{c}{ Clearance\% } \\
& Luteolin & Fe complex & Mg complex & Cu complex & Ca complex & Fe salt & Mg salt & Cu salt & Ca salt \\
\hline 1.0 & 80.27 & 89.69 & 73.44 & 94.63 & 79.46 & 29.81 & 7.00 & 39.39 & 5.70 \\
0.1 & 69.66 & 72.66 & 73.30 & 86.54 & 76.02 & 16.9 & -0.84 & 7.83 & 4.79 \\
0.01 & 18.34 & 15.03 & 31.21 & 36.72 & 34.65 & 7.7 & -1.29 & 0.08 & 4.26 \\
\hline
\end{tabular}

around the above two retention times using automatic detection software, two small protuberances, which were similar in shape to the two chromatographic peaks in the $20 \%$ formic acid-methanol sample, were present in the baseline by manual examination, suggesting the presence of similar compounds at $55.249 \mathrm{~min}$ and $56.273 \mathrm{~min}$.
We noticed that luteolin-metal complexes were slightly dissolved in methanol but completely dissolved in $20 \%$ formic acid-methanol into ligands and corresponding metal ions (data not shown). The chromatographic peak area of both L. japonica and Huangshan wild chrysanthemum decoction in $20 \%$ formic acid-methanol samples was higher 

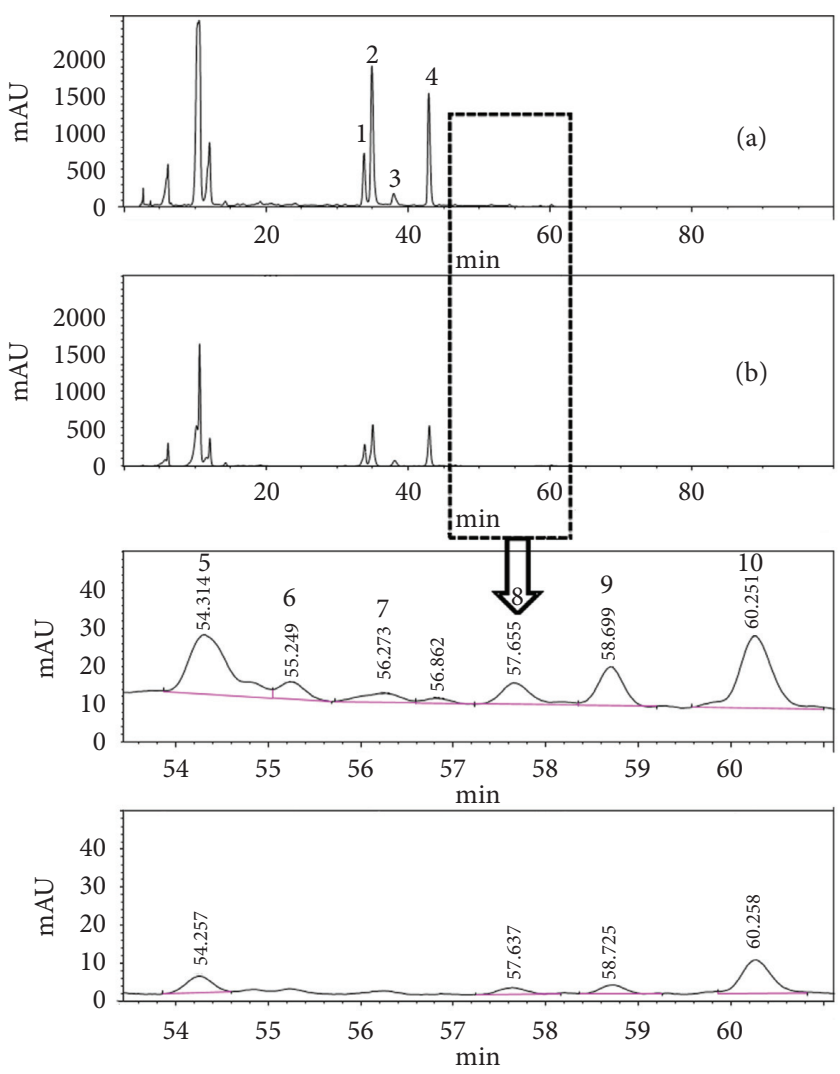

Figure 5: The chromatographic profiles of the $20 \%$ formic acid-methanol extract (a) and methanol extract (b) of $L$. japonica decoction.

TABLE 2: Retention time and peak area of L. japonica decoction $(\bar{X} \pm S, n=3)$.

\begin{tabular}{lccccc}
\hline \multirow{2}{*}{ No. } & \multicolumn{2}{c}{$20 \%$ formic acid-methanol sample } & \multicolumn{2}{c}{ Methanol sample } & \multirow{2}{*}{ Increase in peak area (\%) } \\
& Retention time & Peak area & Retention time & Peak area & 129.94 \\
2 & 33.806 & $18562.99 \pm 1078.91$ & 33.898 & $8072.82 \pm 487.77$ & 233.40 \\
3 & 34.925 & $55410.47 \pm 3220.53$ & 35.055 & $16619.99 \pm 1004.20$ & 220.08 \\
4 & 38.134 & $8282.763 \pm 481.41$ & 38.186 & $2587.756 \pm 156.35$ & 177.31 \\
5 & 42.941 & $37570.57 \pm 2183.65$ & 42.982 & $13548.2 \pm 818.59$ & 449.17 \\
6 & 54.314 & $486.37 \pm 28.27$ & 54.257 & $88.5658 \pm 5.35$ & - \\
7 & 55.249 & $91.60 \pm 5.32$ & Not detected & Not detected & - \\
8 & 56.273 & $73.73 \pm 4.29$ & Not detected & Not detected & 326.48 \\
9 & 57.655 & $141.49 \pm 8.22$ & 57.637 & $33.18 \pm 2.00$ & 351.95 \\
10 & 58.699 & $195.47 \pm 11.36$ & 58.725 & $43.25 \pm 2.61$ & 135.60 \\
\hline
\end{tabular}

than that in the methanol samples (Tables 2 and 3). The chromatographic peak of luteolin in L. japonica decoction in $20 \%$ formic acid-methanol samples increased 1.36 fold, while that of Huangshan wild chrysanthemum decoction increased 1.61 .

\section{Discussion}

Luteolin is one of the important plant metabolites. Due to the presence of carbonyl and hydroxyl groups, luteolin can coordinate metal ions to form complexes, and the metal complexes have shown higher biological effects than free luteolin. Over recent years, many luteolin-metal ion complexes have been synthesized including luteolin complexes with copper
$[18,19]$, cadmium [20], chromium [20, 21], vanadium [22-24], aluminum [24-26], manganese [27], zinc [28, 29], iron (III) $[30,31]$, and rare earth elements [32]. However, these luteolinmetal ion complexes are synthesized in an organic solvent or alkaline aqueous solution. Anna et al. demonstrated by NMR that the energetically favored $\mathrm{Zn}$ chelation sites of the $1: 1$ zincluteolin complexes were $4=\mathrm{O}$ and 5-OH [25]. Rygula et al. [26] showed that, after dissolving in sodium hydroxide, luteolin and $\mathrm{Zn}^{2+}$ mainly coordinate at $3^{\prime}-\mathrm{OH}$ and $4^{\prime}-\mathrm{OH}$ of luteolin in Tris$\mathrm{HCl}$ ( $\mathrm{pH}$ 7.4). Hao et al. [27] detected three reaction products, such as $\left[(\mathrm{Lu}-2 \mathrm{H})^{2-}+\mathrm{Fe}^{3+}+\mathrm{NO}_{3}{ }^{-}+\mathrm{H}^{+}\right], \quad[(\mathrm{Lu}-2 \mathrm{H})$ $\left.{ }^{2-}+\mathrm{Fe}^{3+}+2 \mathrm{CH}_{3} \mathrm{CH}_{2} \mathrm{OH}\right]$, and $[(\mathrm{Lu}-2 \mathrm{H})$ $\left.{ }^{2-}+\mathrm{Fe}^{3+}+\mathrm{NO}_{3}{ }^{-}+2 \mathrm{CH}_{3} \mathrm{CH}_{2}-\mathrm{OH}+\mathrm{H}^{+}\right]$, by ESI-TOF-MS when luteolin reacted with $\mathrm{Fe}\left(\mathrm{NO}_{3}\right)_{3} \cdot 9 \mathrm{H}_{2} \mathrm{O}$ in ethanol; 

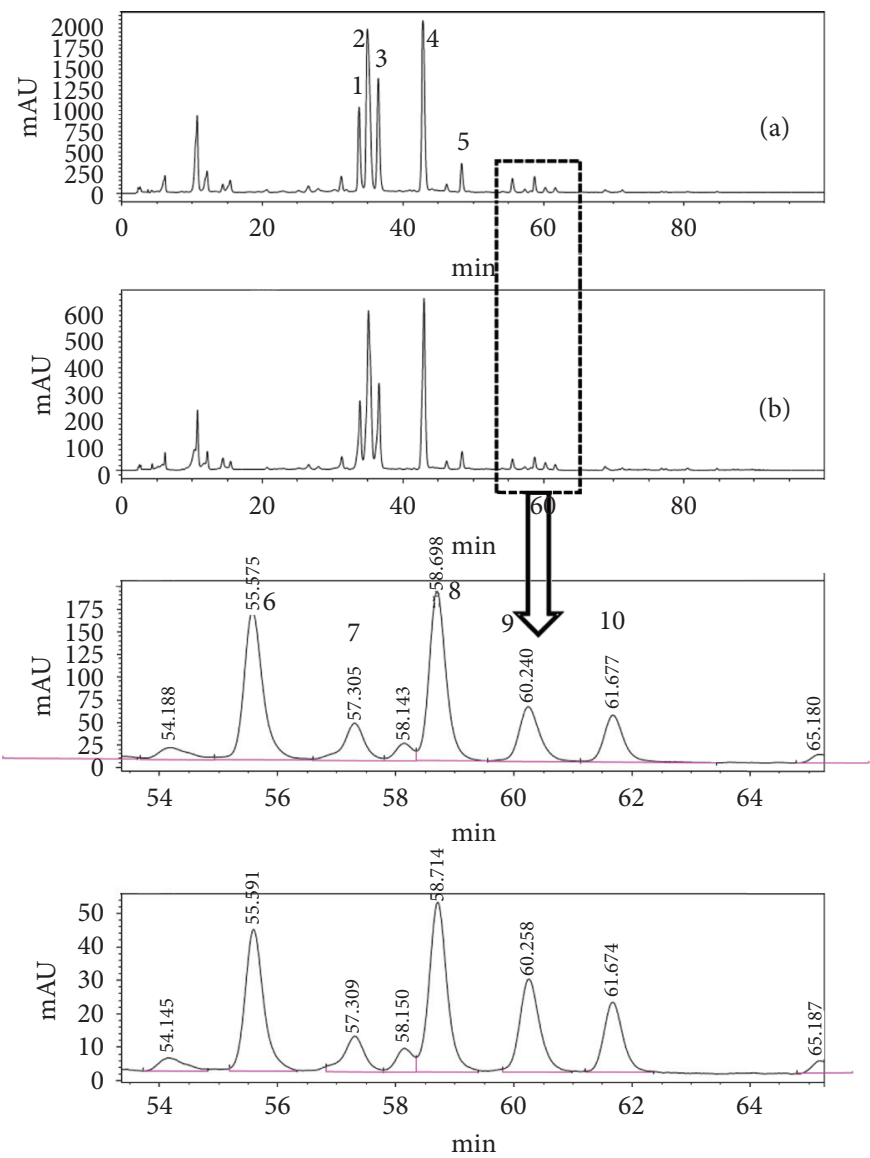

Figure 6: The chromatographic profiles of the $20 \%$ formic acid-methanol extract (a) and methanol extract (b) of Huangshan wild chrysanthemum decoction.

TABLE 3: Retention time and peak area of Huangshan wild chrysanthemum decoction $(\bar{X} \pm S, n=3)$.

\begin{tabular}{lccccc}
\hline \multirow{2}{*}{ No. } & \multicolumn{2}{c}{$20 \%$ formic acid-methanol sample } & \multicolumn{2}{c}{ Methanol sample } & Increase in peak area (\%) \\
& Retention time & Peak area & Retention time & Peak area & 223.95 \\
2 & 33.77 & $25286.71 \pm 1699.06$ & 33.908 & $7805.70 \pm 689.08$ & 199.51 \\
3 & 34.937 & $72512.27 \pm 4872.24$ & 35.089 & $24210.14 \pm 2137.26$ & 256.65 \\
4 & 36.446 & $34578.05 \pm 2323.36$ & 36.556 & $9695.21 \pm 855.89$ & 206.49 \\
5 & 42.804 & $60551.63 \pm 4068.58$ & 42.970 & $19756.52 \pm 744.10$ & 345.18 \\
6 & 48.329 & $7949.30 \pm 534.13$ & 48.391 & $1785.63 \pm 157.63$ & 308.21 \\
7 & 55.575 & $3824.70 \pm 256.99$ & 55.591 & $936.94 \pm 82.71$ & 379.69 \\
8 & 57.305 & $1077.09 \pm 72.37$ & 57.309 & $224.54 \pm 19.82$ & 255.18 \\
9 & 58.698 & $3877.06 \pm 260.51$ & 58.714 & $1091.58 \pm 96.36$ & 131.61 \\
10 & 60.240 (luteolin) & $1538.59 \pm 103.38$ & $60.258($ luteolin) & $664.29 \pm 58.64$ & $479.94 \pm 42.37$ \\
\hline
\end{tabular}

moreover, these reaction products enhanced the clearance of DPPH free radicals. As most edible and medicinal plants are decocted in water before use, the current study used distilled water as solvent to investigate the formation of complexes between luteolin and metal ions in plant aqueous extracts. Our study demonstrated that luteolin and $\mathrm{Zn}^{2+}$ did not form complexes in distilled water, while the reaction products of luteolin and $\mathrm{Fe}^{3+}$ lowered the clearance of DPPH free radicals by luteolin, suggesting that reaction solvent affects the ability of luteolin to complex with metal ions, chelation sites, and activities of complexes.
In analyzing methanol and $20 \%$ formic acid-methanol samples of L. japonica and Huangshan wild chrysanthemum decoction, we took great efforts to minimize variations in weighing and extracting the medicinal herbs and divided a decoction into two parts equivalent in volume which was then spun dry and dissolved in solutions. Furthermore, filtration was performed at $95^{\circ} \mathrm{C}$ in the waterbath to prevent precipitation of compounds due to decrease in filtrate temperature, leading to great variations in results. HPLC revealed that the area of chromatographic peaks for luteolin and other compounds increased to various extents in 
L. japonica and Huangshan wild chrysanthemum decoction in $20 \%$ formic acid-methanol samples. Though L. japonica and Huangshan wild chrysanthemum contain, apart from luteolin, glycosides from luteolin complexation with carbohydrates, we used moderate to strong acid, formic acid, which was of relatively low concentration in the solution, and the dissolution condition was relatively mild; consequently, the luteolin peak area in $20 \%$ formic acid-methanol solution markedly increased. Increased luteolin content was unlikely caused by increased luteolin-glycoside complexes but more likely due to dissolution of luteolin-metal complexes in the presence of formic acid. Moreover, the amplitude of increase in the luteolin peak area of the decoctions of the two plants was different in $20 \%$ formic acid-methanol, which may be due to the difference in the types and contents of metal ions in the two plants.

\section{Conclusion}

The current study has demonstrated that, apart from organic compounds and metal ions, complexes formed from organic compounds and metal ions are also present in the decoction of a plant. These findings suggest that investigators should also characterize and study the biological significance of organic compound-metal ion complexes in plants.

\section{Data Availability}

The datasets used and/or analyzed during the current study are available from the corresponding author upon request.

\section{Conflicts of Interest}

The authors declare that they have no conflicts of interest.

\section{Acknowledgments}

This work was supported by grants obtained from Jiangxi Youth Science Fund Project (20122BAB215017) and the Jiangxi Science and Technology Normal University Undergraduate Research Fund Project (20181204073).

\section{References}

[1] "Antihyperglycemic effect of Passiflora glandulosa cav. fruit rinds flour in streptozotocin-induced diabetic mice," Asian Pacific Journal of Tropical Medicine, vol. 11, no. 9, pp. 510-517, 2018.

[2] M. Chatatikun, T. Yamauchi, K. Yamasaki, A. Chiabchalard, and S. Aiba, "Phyllanthus acidus (L.) Skeels and Rhinacanthus nasutus (L.) Kurz leaf extracts suppress melanogenesis in normal human epidermal melanocytes and reconstitutive skin culture," Asian Pacific Journal of Tropical Medicine, vol. 12, no. 3, pp. 98-105, 2019.

[3] J. Rybak and L. Ruzik, "Application of chromatography and mass spectrometry to the characterization of cobalt, copper, manganese and molybdenum in Morinda citrifolia," Journal of Chromatography A, vol. 1281, pp. 19-25, 2013.

[4] J. Wojcieszek, P. Kwiatkowski, and L. Ruzik, "Speciation analysis and bioaccessibility evaluation of trace elements in goji berries (Lycium Barbarum, L.)," Journal of Chromatography A, vol. 1492, p. 70, 2017.

[5] G. Weber and P. Konieczyński, "Speciation of Mg, Mn and Zn in extracts of medicinal plants," Analytical and Bioanalytical Chemistry, vol. 375, no. 8, pp. 1067-1073, 2003.

[6] F. Liu, F. Pei, A. M. Mariga, L. Gao, G. Chen, and L. Zhao, "Separation and speciation analysis of zinc from Flammulina velutipes," Journal of Food and Drug Analysis, vol. 23, no. 4, pp. 630-635, 2015.

[7] M. Lopez-Lazaro, "Distribution and biological activities of the flavonoid luteolin," Mini Reviews in Medicinal Chemistry, vol. 9, no. 1, 2009.

[8] K. H. Miean and S. Mohamed, "Flavonoid (myricetin, quercetin, kaempferol, luteolin, and apigenin) content of edible tropical plants," Journal of Agricultural and Food Chemistry, vol. 49, no. 6, pp. 3106-3112, 2001.

[9] G. Seelinger, I. Merfort, and C. Schempp, "Anti-oxidant, anti-inflammatory and anti-allergic activities of luteolin," Planta Medica, vol. 74, no. 14, pp. 1667-1677, 2008.

[10] U. zgen, A. Mavi, Z. Terzi et al., "Relationship between chemical structure and antioxidant activity of luteolin and its glycosides isolated from T. Sipyleus subsp. sipyleus var. sipyleus," Records of Natural Products, vol. 13, no. 5, pp. 1-12, 2010.

[11] T. Zhang, Y. Qiu, Q. Luo et al., "The mechanism by which luteolin disrupts the cytoplasmic membrane of methicillin-resistant Staphylococcus aureus," The Journal of Physical Chemistry B, vol. 122, no. 4, p. 1427, 2018.

[12] X. Lin, S. Weiheng, J. Jun et al., "Identification of luteolin as enterovirus 71 and coxsackievirus A16 inhibitors through reporter viruses and cell viability-based screening," Viruses, vol. 6, no. 7, pp. 2778-2795, 2014.

[13] X. Lu, Y. Li, X. Li, and H. A. Aisa, "Luteolin induces apoptosis in vitro through suppressing the MAPK and PI3K signaling pathways in gastric cancer," Oncology Letters, vol. 14, no. 2, pp. 1993-2000, 2017.

[14] K. A. Kang, M. J. Piao, Y. S. Ryu et al., "Luteolin induces apoptotic cell death via antioxidant activity in human colon cancer cells," International Journal of Oncology, vol. 51, no. 4, pp. 1169-1178, 2017.

[15] H. Qiao, X. Zhang, C. Zhu et al., "Luteolin downregulates TLR4, TLR5, NF- $\kappa$ B and p-p38MAPK expression, upregulates the p-ERK expression, and protects rat brains against focal ischemia," Brain Research, vol. 1448, pp. 71-81, 2012.

[16] X. Wu, T. Xu, D. Li et al., "ERK/PP1a/PLB/SERCA2a and JNK pathways are involved in luteolin-mediated protection of rat hearts and cardiomyocytes following ischemia/reperfusion," PLoS One, vol. 8, no. 12, Article ID e82957, 2013.

[17] M. D. Catarino, A. M. S. Silva, S. C. Saraiva et al., "Characterization of phenolic constituents and evaluation of antioxidant properties of leaves and stems of Eriocephalus africanus," Arabian Journal of Chemistry, vol. 196, pp. 62-69, 2015.

[18] S. Niu, B. Han, W. Cao, and S. Zhang, "Sensitive DNA biosensor improved by Luteolin copper(II) as indicator based on silver nanoparticles and carbon nanotubes modified electrode," Analytica Chimica Acta, vol. 651, no. 1, pp. 42-47, 2009.

[19] S. Shuyun, Z. Yuping, C. Xiaoqin et al., "Investigation of flavonoids bearing different substituents on ring $\mathrm{C}$ and their cu2+ complex binding with bovine serum albumin: structure-affinity relationship aspects," Journal of Agricultural and Food Chemistry, vol. 59, no. 19, pp. 10761-10769, 2011.

[20] L.-G. Gao, H. Wang, X.-L. Song, and W. Cao, "Research on the chelation between luteolin and $\mathrm{Cr}$ (III) ion through 
infrared spectroscopy, UV-vis spectrum and theoretical calculations," Journal of Molecular Structure, vol. 1034, no. 6, pp. 386-391, 2013.

[21] W. Cao, "Luteolin and luteolin-Cr (III) complexes: antioxidation and reaction mechanism with hydrogen peroxide radical," Chinese Journal of Inorganic Chemistry, vol. 31, no. 11, pp. 2229-2235, 2015.

[22] S. Roy, S. Mallick, T. Chakraborty et al., "Synthesis, characterisation and antioxidant activity of luteolin-vanadium (II) complex," Food Chemistry, vol. 173, pp. 1172-1178, 2015.

[23] L. Naso, V. R. Martínez, L. Lezama et al., "Antioxidant, anticancer activities and mechanistic studies of the flavone glycoside diosmin and its oxidovanadium(IV) complex. Interactions with bovine serum albumin," Bioorganic \& $M e$ dicinal Chemistry, vol. 24, no. 18, pp. 4108-4119, 2016.

[24] L. G. Naso, L. Lezama, M. Valcarcel et al., "Bovine serum albumin binding, antioxidant and anticancer properties of an oxidovanadium(IV) complex with luteolin," Journal of Inorganic Biochemistry, vol. 157, no. 5, pp. 80-93, 2016.

[25] A. Anna, C. Catia, M. Costanza et al., "Complexation of apigenin and luteolin in weld lake: a DFT/TDDFT investigation," Physical Chemistry Chemical Physics Pccp, vol. 12, no. 25, pp. 6672-6684, 2010.

[26] A. Rygula, T. P. Wrobel, J. Szklarzewicz, and M. Baranska, "Raman and UV-vis spectroscopy studies on luteolin-Al (III) complexes," Vibrational Spectroscopy, vol. 64, no. 8, pp. 21-26, 2013.

[27] D. Hao, X. Yang, J. He et al., "Enhanced antioxidant activity, antibacterial activity and hypoglycemic effect of luteolin by complexation with manganese(II) and its inhibition kinetics on xanthine oxidase," Rsc Advances, vol. 7, no. 84, pp. 53385-53395, 2017.

[28] P. Alexandra, M. Gloria, L. Christina et al., "Understanding zinc(II) chelation with quercetin and luteolin: a combined NMR and theoretical study," Journal of Physical Chemistry B, vol. 119, no. 1, pp. 83-95, 2015.

[29] B. Xiao, H. Wang, X. Zhao, and Y. Li, "Selective recognition of luteolin and quercetin based on the specific interaction of ortho-dihydroxy substituents with a zinc (ii) complex," $A n$ alytical Methods, vol. 6, no. 9, pp. 2894-2899, 2014.

[30] A.-H. Yang, X.-Y. Shi, X. Li et al., "Spectroscopic and electrochemical studies on the evaluation of the radical scavenging activities of luteolin by chelating iron," Rsc Advances, vol. 4, no. 48, pp. 25227-25233, 2014.

[31] R. Jun, M. Sheng, C. E. Lekka et al., "Complexation of flavonoids with iron: structure and optical signatures," Journal of Physical Chemistry B, vol. 112, no. 6, pp. 1845-1850, 2008.

[32] J. Li, L. Wang, H. Bai, B. Yang, and H. Yang, "Synthesis, characterization, and anti-inflammatory activities of rare earth metal complexes of luteolin," Medicinal Chemistry Research, vol. 20, no. 1, pp. 88-92, 2011. 